\title{
Retinal Vascular Occlusion and SARS-CoV-2 Vaccination
}

\section{Stela Vujosevic ( $\square$ stela.vujosevic@gmail.com )}

University of Milan: Universita degli Studi di Milano https://orcid.org/0000-0001-6773-9967

\section{Celeste Limoli}

University of Milan: Universita degli Studi di Milano

\section{Simona Romano}

Multimedica Group: MultiMedica SpA

Lucia Vitale

Multimedica Group: MultiMedica SpA

\section{Edoardo Villani}

Universita degli Studi di Milano

\section{Paolo Nucci}

University of Milan: Universita degli Studi di Milano

\section{Research Article}

Keywords: Retinal vascular occlusion, Retinal imaging, COVID-19, COVID-19 vaccination

Posted Date: February 22nd, 2022

DOI: https://doi.org/10.21203/rs.3.rs-1346316/v1

License: (c) (i) This work is licensed under a Creative Commons Attribution 4.0 International License. Read Full License 


\section{Abstract}

Purpose: to assess the clinical and retinal imaging features of patients in whom retinal vascular occlusion had developed in temporal associations with Covid-19 vaccination.

Methods: in this retrospective case series, all consecutive adult patients with new onset retinal vascular occlusion (VO) within 6 weeks of vaccination against Covid-19 were included in the study between May $1^{\text {st }}$ and October $31^{\text {st }} 2021$. All patients had a systemic medical health assessment, full ophthalmic evaluation and complete fundus imaging.

Results: Fifteen cases of retinal vascular occlusion (14 patients) after Covid-19 vaccinations were identified. The median time between vaccination and symptoms onset was 14 days (range 7-42 days). VO included central retinal vein occlusion (CRVO) in 5 (33.3\%) eyes, hemiretinal vein occlusion (HRVO) in 2 (13.3\%) eyes, branch retinal vein occlusion (BRVO) in 7 (46.6\%) eyes, and 1 (6.6\%) eye with central retinal artery occlusion (CRAO). The mean BCVA was 20/55 with a range of 20/20 to 20/200. Eleven of 15 eyes (73.3\%) had visual acuity improvement after intravitreal treatment at 60-90 days (range, 45-105 days) from the presentation. Number of new cases of VO was higher in the considered period compared to the equivalent 6 months' period in $2019(1.17 \%$ vs $0.52 \%$ respectively; $P=0.0134)$.

Conclusions: Retinal vascular occlusion with different grades of severity are reported in temporal association with Covid-19 vaccination. Prompt diagnosis with appropriate treatment may provide patients with better visual acuity outcome. The exact pathogenic mechanism needs to be further studied. No certain causal relationship can be established from this case series.

\section{Key Messages}

- Unusual thrombotic systemic event after receiving Covid-19 vaccines have been reported in literature.

- In this retrospective case series, we present 15 eyes (14 patients) with retinal Vo presenting from 1 to 6 weeks after receiving a dose of the Covid-19 vaccine, either mRNA vaccines or vector ones. The condition occurred throughout the post-vaccination period, but it had occurred more often within 2-6 weeks after the first dose of ChAd0x1, whereas within 1 week after the second dose of BNT162b2.

- Covid-19 vaccinations could result in the rare development of retinal Vo, however no certain causal relationship can be established from this study design.

\section{Introduction}

The development of vaccines against Severe Acute Respiratory Syndrome Coronavirus 2 (SARS-CoV-2) infection has been shown to be the most important countermeasure to curb Covid-19 (coronavirus disease) pandemic. Vaccines have been reported to protect against severe illness from SARS-CoV-2 infection ${ }^{1-5}$. Thus far, four types of Covid-19 vaccines have been approved by the European Medicines Agency: mRNA vaccines, including BNT162b2, Pfizer-BioNTech, and mRNA-1273, Moderna; vector vaccines, including ChAdOx1 nCoV-19/ AZD1222, Oxford-AstraZeneca ${ }^{1-5}$ and Ad26.COV2, Janssen Johnson \& Johnson.

The mass vaccination campaign against SARS-CoV-2 started in Italy on the $27^{\text {th }}$ of December 2020. As of October 2021, the Italian Ministry of Health and Prevention announced that over 45 millions of people (83,5\% of the population) had completed the recommended scheme and were fully vaccinated ${ }^{6}$. The mRNA vaccination is administered in 2-dose series separated by 3 weeks (21 days), the ChAdOx1 in 2-dose series separated by 8-12 weeks, whereas Ad26.COV2 has a single-dose regimen ${ }^{6}$.

Given the scale of the current vaccination program, several rare ocular adverse events related to vaccines have been reported and their potential manifestations constitute a safety concern 7 .

In this single-centre case series, we hereby define the longitudinal characteristics of patients with retinal vascular occlusion (VO) in the temporal context of SARS-CoV-2 vaccinations, focusing on the time lapse between vaccination and disease onset, clinical and imaging features, and short-term outcomes. 
A report of such ocular adverse events is timely and would be beneficial to design and implement protocols for close monitoring of patients that may be at higher risk.

\section{Methods}

All consecutive adult patients with new onset retinal VO within 6 weeks of vaccination against Covid-19 who presented to the Medical Retina \& Imaging Service at the University Eye Clinic San Giuseppe Hospital, Milan, Italy, between 1 May 2021 and 31 October 2021, were included in this retrospective case series study.

The diagnosis of $\mathrm{VO}$ was based on a comprehensive ophthalmic examination, best corrected visual acuity determination (BCVA); slit lamp evaluation, intraocular pressure (IOP), dilated funduscopy, optical coherence tomography (OCT) and OCT angiography (OCT-A) (Heidelberg Spectralis HRA-OCT; Heidelberg Engineering, Heidelberg, Germany), ultra-wide field fundus color photo and fluorescein angiography (FFA) (Optos California, Optos PLC, Dunfermline, United Kingdom).

Retinal vein occlusion (RVO) was classified as central retinal vein occlusion (CRVO), hemiretinal vein occlusion (HRVO), and branch retinal vein occlusion (BRVO), according to the definition used by Hayreh ${ }^{8}$. A meticulous diagnostic work up excluded any underlying hematologic or vasculitis disease with possible influence on the onset of RVO. No other concurrent ocular conditions were present at the time of the diagnosis. Patients provided proof of a negative Covid-19 test that met performance standards, as recorded on the charts $^{9}$.

Vaccination data by November 2021 including the administered number of vaccine doses, types and presence of ocular adverse event post Covid-19 vaccination in Italy are provided in Table 17,10.

To investigate the potential change in the new cases of VO related to Covid-19 vaccines among patients presenting to our Medical Retina \& Imaging Service, we compared the incidence of VO in the 6 months' inclusion period (May the $1^{\text {st }} 2021$ - October $31^{\text {st }}$ 2021) to the equivalent period prior to the onset of Covid-19 pandemic (May $1^{\text {st }} 2019-31^{\text {st }}$ October 2019) .

The study was approved by the ethics committee of IRCCS MultiMedica and adhered to the tenets of the declaration of Helsinki. Each patient gave informed consent for use of data.

Statistical Analysis. Categorical variables were reported with number and percentage, and continuous variables were summarized with mean and range. Univariate comparison between the two groups of VO patients was performed using the Fisher's exact test. A $p$ value 0.05 was considered significant. The data analysis was performed using SPSS Statistics version 28 (IBM).

\section{Results}

Fifteen cases of VO (14 patients) in temporal association with SARS- CoV-2 vaccinations (BNT162b2 $n=8$, ChAdOx1 $n=6$, Ad26.COV2 $n=1$ ) were identified.

Six cases (40\%) occurred after the first ChAdOx1 dose, 8 cases (53.3\%) were noted following the second BNT162b2 dose, and 1 case (6.6\%) after Ad26.COV2. The median time between vaccination and symptoms onset was 14 days (range 7-42 days).

Characteristics of all 14 patients are presented in Table 2.

In the present case series, women were affected slightly more often than men (9 (64.2\%) versus $5(35.7 \%)$, respectively); the median age was 77 years (range, 40 to 96 ). Nine patients (64.2\%) met the criteria for classic risk factors for RVO. More specifically, 7 (50\%) patients had systemic hypertension (HTN), 4 (28.5\%) had hyperlipidemia (HL), 3 (21.4\%) had diabetes mellitus type 2 (T2DM), where $6(42.8 \%)$ patients had 2 of these risk factors and $1(7.1 \%)$ had all 3 . One patient $(7.1 \%)$ had a previous ocular history of BRVO in the fellow eye. Four patients (28.5\%) had negative medical history.

Unilateral acute vision loss was the most common presenting symptom; 1 patient (7.1\%) presented with bilateral vision loss. The mean BCVA was $20 / 55$ with a range of $20 / 20$ to $20 / 200$. The most severely affected eyes (patients $3,4,8,12$ ) had severe visual acuity loss with BCVA of 20/200 at the time of diagnosis and showed limited improvement at latest follow-up (range, 45-105 days).

Page $3 / 9$ 
A spectrum of severity of clinical findings found at presentation is outlined in Table 2.

VO included CRVO in $5(33.3 \%)$ eyes, HRVO in $2(13.3 \%)$ eyes, BRVO in $7(46.6 \%)$ eyes, and $1(6.6 \%)$ eye with central retinal artery occlusion (CRAO).

Treatment included administration of intravitreal anti-vascular endothelial growth factor (VEGF) in 1 (6.6\%) eye, the use of a sustained-release intravitreal dexamethasone implant in 7 (46.6\%) eyes, whereas $2(13.3 \%)$ eyes required retinal laser photocoagulation due to extensive ischemia, not in association with anti-VEGF injections. Overall, 11 eyes (73.3\%) had visual acuity improvement at 2-3 months (range, 45-105 days) from the presentation, however 4 (26.6\%) eyes that did not achieve any BCVA restoration from the presentation. Although the follow-up was relatively short, there has been no recurrence of RVO. Of 2470 cases visited at the Medical retina service from May $1^{\text {st }}$ to October $31^{\text {st }} 2021$, there were 29 cases of VO, including the abovementioned 15 cases temporally related to Covid-19 vaccination, with estimated incidence of $1.17 \%$ cases. In the same temporal interval in 2019, there were 2671 cases of which 14 cases with VO $(0.52 \%)$ visited at the Medical Retina service. The increase in the number of newly diagnosed VO was significantly higher in 2021 vs those in 2019, before the Covid-19 pandemic, $p=0.0134$.

\section{Selected cases}

Patient 10 (Figure 1). A 72-year-old woman presented to the retina clinic reporting blurry vision in the right eye 1 week after receiving the second dose of BNT162b2. She had a known medical history of HTN and HL under medical and pharmacological control. Ocular history was unremarkable. BCVA at presentation was 20/25 and dilated ocular fundus examination revealed retinal hemorrhages in the supero-temporal sector. The OCT revealed cystoid macular edema with central retinal thickness of 431 micron. OCT-A at the level of the superficial capillary plexus (SCP) showed non-perfusion areas in the superotemporal macular region and decreased vascular density, more evident in SCP than in the deep capillary plexus (DCP). FFA showed delay in the perfusion of the supero-temporal branch vein with late leakage. No epiretinal or epipapillary neovascularizations were identified. The treatment consisted of 1 intravitreal injection of dexamethasone implant. At the follow up visit, the macular edema resolved, and visual acuity improved to $20 / 20$.

Patient 13 (Figure 2). A 65-year-old woman presented to the retina clinic complaining of decreased and blurred vision in her right eye, 1 week after receiving Ad26.COV2 vaccine. The patient's medical history revealed T2DM, HTN and HL, well controlled with medications. No other systemic diseases were reported. BCVA at baseline was 20/40. Anterior segment documented pseudophakia, and IOP within normal limits. Fundus examination revealed numerous flame-shaped hemorrhages in all 4 quadrants, optic disc edema and macular edema. No cells in the vitreous were detected. FFA showed a delay in the central retinal vein perfusion, no significant ischemia in the periphery and late hyperfluorescence due to the intraretinal dye leakage in the macula and of the optic nerve. Patient was diagnosed with unilateral CRVO in the right eye.

OCT of the macula documented cystoid macular edema with serous neuroretinal detachment and thickening of the central retina up to 621 microns. OCT-A at the level of the SCP showed vascular tortuosity and marked decrease in vascular perfusion at the level of the DCP. The results of blood tests, including lipid profile, and complete blood count were normal, blood glucose was well controlled and no thrombophilia risk factors were identified, the electrocardiogram and the echocardiography were unremarkable.

The treatment consisted of 1 intravitreal injection of dexamethasone implant. After 3 months, the macular edema resolved but limited improvement in visual acuity over the three months of treatment was observed, with a BCVA of 20/32.

\section{Conclusion}

In this case series, we present 15 eyes (14 patients) with RVO and CRAO presenting from 1 to 6 weeks after receiving a dose of the Covid-19 vaccine, either mRNA vaccines or vector ones. The condition occurred throughout the post-vaccination period, but it had occurred more often within 2-6 weeks after the first dose of ChAdOx1, whereas within 1 week after the second dose of BNT162b2.

The clinical course of the condition had a range in the severity of findings; most patients responded well to therapy or recovered spontaneously, and visual acuity was preserved. Four eyes (26.6\%) with an occlusion of either central artery or vein, and retinal vein proximal to the macula showed poor outcomes, with BCVA of 20/200 after 3 months of follow-up. 
RVOs are a group of vascular disorders characterized by an impaired venous drainage from the retinal circulation. Although the exact patho-physiology of RVO remains elusive, it is well established that the retinal microvasculature impairment and the Virchow's triad, which consists of hypercoagulability, venous stasis, endothelial injury, may predispose to the development of vein occlusions $^{12}$. Advancing age and systemic vascular disorders, including HTN, HL, and metabolic disease such as T2DM are strongly associated with RVO. The most common ocular risk factor associated with RVO is glaucoma. In young patients with no obvious RVO etiology, it is recommended to consider thrombophilic factors, such as hyperhomocysteinemia ${ }^{12}$.

CRAO is an ophthalmic emergency caused by the sudden blockage of the central retinal artery. Although it shares the same cardiovascular risk factors as RVO, it is considered analogous to a cerebral stroke or transient ischemic attacks and, therefore, it requires a multidisciplinary approach with stroke expertise ${ }^{13}$.

Our cohort of patients notably included 13 patients (92.9\%) older than 65 years old, except for 1 patient (7.1\%) that was 40 years old. In our case series, 9 patients (64.3\%) had the classic risk factors for RVO, defined as the presence of HTN, HL and/or T2DM. The 40year-old patient had hyperhomocysteinemia, already reported as a risk factor for $\mathrm{RVO}^{12}$.

The report of ocular adverse events occurred after Covid-19 vaccines is published on a monthly basis by AIFA in Italy ${ }^{10}$ and is presented in Table 1; the incidence rates sum up to 1.716/99.797.303 doses of vaccine, i.e. an overall rate of 1.70 cases/10 5 vaccine doses. Therefore, the occurrence of ocular adverse events after SARS- CoV-2 immunization is rare.

In this study, we have found a statistically significant increase in new cases of Vo between May and the end of October 2021 compared to the numbers of the same 6 months' interval in 2019. Therefore, this observation could foster the temporal association between Covid-19 vaccination and VO to explain the increase in the number of patients presenting with VO in the short period of time noted above. However, we need to acknowledge that this study was not powered to examine incidences of vascular ophthalmic events after receiving Covid-19 vaccination.

Different ophthalmic manifestations after Covid-19 vaccinations have been reported, including facial nerve palsy, abducens nerve palsy, superior ophthalmic vein thrombosis, corneal graft rejection, uveitis, anterior segment inflammation, central serous chorioretinopathy, Vogt-Koyanagi-Harada disease reactivation, and onset of Graves' disease ${ }^{14}$. A recent case series study has described different ocular adverse events, including scleritis, episcleritis and acute maculopathies, occurred 5.2 days after the inactivated Covid-19 vaccine by Sinopharm's China National Biotec Group ${ }^{15}$. These ocular findings seem to overlap with ophthalmic manifestations induced by Covid-19 itself, implying a common pathogenetic pathway between Sars-Cov-2 virus and vaccinemediated immune response ${ }^{14}$. It has been suggested that the pathogenesis of abnormalities in the retina subsequent to Covid-19 vaccinations, regardless they are adenovirus vector or mRNA vaccines, could be explained by molecular mimicry, where the spike antigen or other viral epitopes share structural similarities with self-antigens leading to an immunological self-tolerance break and an autoimmune response, and by type II or type IV hypersensitivity reactions ${ }^{16}$.

Retinal vascular adverse events after Covid-19 vaccinations appear to be rare ${ }^{12}$. Only, 2 single case reports have been available in the literature. One study documented a single patient with CRVO occurring immediately after the 2nd vaccination with an mRNAbased vaccine ${ }^{17}$. In the other report, a healthy 55 -year-old woman presented with bilateral superior ophthalmic vein thrombosis after 10 days from her first vaccine dose against SARS-CoV-2- ChAdOx $1 \mathrm{nCoV}-19$, accompanied by ischaemic stroke, and immune thrombocytopenia ${ }^{18}$.

Several studies have reported single cases or series of patients who developed unusual thrombotic systemic event after receiving Covid-19 vaccines, including ChAdOx1 nCoV-19, Ad26.COV2.S ${ }^{19-28}$. The condition is described as Covid-19 vaccine-related thrombosis and thrombocytopenia, namely thrombosis with thrombocytopenia syndrome (TTS).

Three separate case series have described patients who developed TTS after ChAdOx1 vaccination ${ }^{19-21}$. TTS, which clinically simulate heparin-induced thrombocytopenia, is mediated by platelet-activating antibodies against Platelet Factor 4 (PF4) ${ }^{22}$ and, interestingly, is more frequently observed following the first dose of ChAdOx $1^{23-26}$ suggesting that any underlying mechanism might be predominant after the first dose ${ }^{27}$. Furthermore, it has been noted that TTS manifest at 1 to 4 weeks period post-vaccination, 
which corresponds to the time for mounting a secondary antibody response ${ }^{22,26}$. Similar haematological findings have been reported after Ad26.COV2 vaccination, that is an adenoviral-based vaccine like the ChAdOX $1^{28}$.

To investigate the association between Covid-19 vaccines and hematological and vascular adverse events, a Scottish national population-based prospective cohort study has been conducted ${ }^{29}$. Positive associations were seen between the first-dose of ChAdOX1 and idiopathic thrombocytopenic purpura as well as venous, arterial thromboembolic and hemorrhagic events ${ }^{29}$. BNT162b2, by contrast, did not show a statistically significant association with the aforementioned adverse events ${ }^{29}$.

Interestingly, our findings of RVO occurrence after the first dose of ChAdOx1 are consistent with the results of these studies. Specifically, it has been observed that the retinal vascular adverse events occurred more often within 2-6 weeks after the first dose of ChAd0x1. Therefore, it may be tempting to suggest a common pathogenic pathway of these thromboembolic events, linking the interaction of adenoviral-based vaccine vector versus SARS-CoV-2 with PF4 and other specific host proteins and the contribution to rare adverse events like RVO.

With regards to mRNA based vaccines, it remains still unknown the pathogenic pathway underlying the observed vascular adverse events. Further studies are needed to investigate it and if there is any common pathogenic mechanism already described for ChAdOx1.

To the best of our knowledge, this is the first case series to report the temporal association between Covid-19 vaccination and VO. It presumably related to SARS-CoV-2 vaccination is an entity that apparently shares several features with the typical VO.

In this context, the pathogenic mechanism of $\mathrm{VO}$ after Covid-19 vaccination has not been elucidated and it is important to emphasize that no certain causality can be established from this case-series.

Finally, one important issue concerns the presumable time period of cause-effect relationship between vaccination and RVO. In this case series, this interval was set to 6 weeks, in reasonable accordance with available data and established knowledge linking vaccines with vascular or autoimmune conditions ${ }^{30}$.

Considering the massive rollout vaccination campaign and the well-established excellent safety profile related to vaccines, it is important to highlight the very low incidence of reported adverse events in the literature. Consequently, Covid-19 vaccination should be strongly encouraged, having shown to be one of the most effective means to reduce the risk of getting and spreading the virus.

In conclusion, retinal vascular occlusion can be considered a rare manifestation of the spectrum of ophthalmic complications after Covid-19 vaccination. Patients with pre-existing cardiovascular risk factors seem to be more likely to develop this complication, however further studies with more data are warranted to draw final conclusions about eventual association between RVO/CRAO and Covid-19 vaccinations.

Therefore, physicians should consider RVO/CRAO if patients present with vision loss within 6 weeks from Covid-19 vaccination. Prompt diagnosis and quick initiation of the appropriate therapy may provide patients with a better visual outcome.

\section{Declarations}

Acknowledgments: None

Financial support: None

Conflict of interest:

Stela Vujosevic: Abbvie-Allergan, Apellis, Bayer, Novartis, Roche SPA: Advisory Board participation. None relevant to this work.

Simona Romano: none

Lucia Vitale: none

Celeste Limoli: none

Page $6 / 9$ 
Edoardo Villani: Alfa Intes (S); Allergan (C,L,S); Bruschettini (L); FB Vision (C,L); OffHealth (S); Oftagest (L); Santen (C,L); Servimed

(C); Sooft (L); Shire (C); Thea (L); Visufarma (L)

C: Consultant/Advisor; L: Lecture fees; S: Grant support (AAO Financial Disclosure and First-slide Policy 2021)

Paolo Nucci: None

\section{References}

1. Polack FP, Thomas SJ, Kitchin N et al (2020) Safety and efficacy of the BNT162b2 mRNA COVID-19 vaccine. N Engl J Med 383:2603-2615

2. Baden LR, El Sahly HM, Essink B et al (2021) Efficacy and safety of the mRNA-1273 SARS-CoV-2 vaccine. N Engl J Med 384:403-416

3. Sadoff J, Gray G, Vandebosch A et al (2021) Safety and efficacy of single-dose Ad26.COV2.S vaccine against Covid-19. N Engl J Med 384(23):2187-2201

4. Voysey M, Clemens SAC, Madhi SA et al (2021) Safety and efficacy of the ChAdOx1 nCoV-19 vaccine (AZD1222) against SARSCoV-2: an interim analysis of four randomised controlled trials in Brazil, South Africa, and the UK. Lancet 397(10269):99-111

5. Gao Q, Bao L, Mao H et al (2020) Development of an inactivated vaccine candidate for SARS-CoV-2. Science 369(6499):77-81

6. Report Vaccini Anti COVID-19, accessed 1 (2021) https://www.governo.it/it/cscovid19/report-vaccini/

7. Sistema di accesso online ai dati delle segnalazioni di sospette reazioni averse registrate nella Rete Nazionale di

Farmacovigilanza, Agenzia Italiana del Farmaco, accessed 1 November 2021, https://bi.aifa.gov.it/SASVisualAnalyticsViewer/VisualAnalyticsViewer_guest.jsp?

reportName=FVG_Intro0_report\&reportPath=/Shared+Data/BI+FARMACOVIGILANZA/Public/Report/\&appSwitcherDisabled=true

8. Hayreh SS (2005) Prevalent misconceptions about acute retinal vascular occlusive disorders. Prog Retin Eye Res 24(4):493519

9. Centers for Disease Control and Prevention. COVID-19 testing overview. Updated March 17, 2021. Accessed November 1 (2021) https://www.cdc.gov/coronavirus/2019-ncov/symptoms-testing/testing.html

10. Rapporto sulla Sorveglianza dei vaccini COVID-19, Agenzia Italiana del Farmaco, accessed 1 November 2021 https://

11. Hirano Y, Suzuki N, Tomiyasu T et al (2021) Multimodal Imaging of Microvascular Abnormalities in Retinal Vein Occlusion. J Clin Med 10:405

12. Zhou JQ, Xu L, Wang S et al (2013) The 10-year incidence and risk factors of retinal vein occlusion: the Beijing eye study. Ophthalmology 120(4):803-808

13. Varma DD, Cugati S, Lee AW, Chen CS (2013) A review of central retinal artery occlusion: clinical presentation and management. Eye (Lond) 27(6):688-697

14. Ng XL, Betzler BK, Testi I et al (2021) Ocular Adverse Events After COVID-19 Vaccination. Ocul Immunol Inflamm 24:1-9

15. Pichi F, Aljneibi S, Neri P et al (2021) Association of Ocular Adverse Events With Inactivated COVID-19 Vaccination in Patients in Abu Dhabi. JAMA Ophthalmol 139(10):1131-1135

16. Jampol LM, Tauscher R, Schwarz HP (2021) COVID-19, COVID-19 Vaccinations, and Subsequent Abnormalities in the Retina: Causation or Coincidence? JAMA Ophthalmol 139(10):1135-1136

17. Bialasiewicz AA, Farah-Diab MS, Mebarki HT (2021) Central retinal vein occlusion occurring immediately after $2 \mathrm{nd}$ dose of mRNA SARS-CoV-2 vaccine. Int Ophthalmol 23:1-4

18. Bayas A, Menacher $M$, Christ $M$ et al (2021) Bilateral superior ophthalmic vein thrombosis, ischaemic stroke, and immune thrombocytopenia after ChAdOx1 nCoV-19 vaccination. Lancet. 1;397(10285):e11

19. Greinacher A, Thiele T, Warkentin TE et al (2021) Thrombotic Thrombocytopenia after ChAdOx1 nCov-19 Vaccination. N. Engl J Med 384(22):2092-2101

20. Schultz NH, Sørvoll IH, Michelsen AE et al (2021) Thrombosis and Thrombocytopenia after ChAdOx1 nCoV-19 Vaccination. N Engl J Med 384(22):2124-2130 
21. Scully M, Singh D, Lown R et al (2021) Pathologic Antibodies to Platelet Factor 4 after ChAdOx1 nCoV-19 Vaccination. N Engl J Med. 10;384(23):2202-2211

22. Baker AT, Boyd RJ, Sarkar D et al (2021) ChAdOx1 interacts with CAR and PF4 with implications for thrombosis with thrombocytopenia syndrome. Sci Adv 7(49):eabl8213

23. Methling K, Völker U, Hentschker $\mathrm{C}$ et al (2021) Towards understanding ChAdOx1 nCov-19 vaccine-induced immune thrombotic thrombocytopenia (VITT). Blood 10.21203/ rs.3.rs-440461/v1

24. Othman M, Baker AT, Gupalo E et al (2021) To clot or not to clot? Ad is the question-Insights on mechanisms related to vaccine induced thrombotic thrombocytopenia. J Thromb Haemost 19:2845-2856

25. Muir KL, Kallam A, Koepsell SA et al (2021) Thrombotic Thrombocytopenia after Ad26.COV2.S vaccination.N. Eng. J. Med.384,1964-1965

26. Greinacher A, Selleng K, Palankar R et al (2021) Insights in ChAdOx1 nCoV-19 vaccine-induced immune thrombotic thrombocytopenia. Blood 138(22):2256-2268

27. Bhuyan P, Medin J, da Silva HG et al (2021) Very rare thrombosis with thrombocytopenia after second AZD1222 dose: A global safety database analysis. Lancet 398:577-578

28. Muir KL, Kallam A, Koepsell SA et al (2021) Thrombotic Thrombocytopenia after Ad26.COV2.S Vaccination. N Engl J Med 384(20):1964-1965

29. Simpson CR, Shi T, Vasileiou E et al (2021) First-dose ChAdOx1 and BNT162b2 COVID-19 vaccines and thrombocytopenic, thromboembolic and hemorrhagic events in Scotland. Nat Med 27(7):1290-1297

30. Vadalà M, Poddighe D, Laurino C et al (2017) Vaccination and autoimmune diseases: is prevention of adverse health effects on the horizon? 8:295-3113

\section{Tables}

Table 1. Vaccination data by November 2021 in Italy and presence of ocular adverse event post COVID-19 vaccination.

\begin{tabular}{lll}
\hline Type of Vaccine & Number of administered doses & Ocular adverse event (total of reported adverse event) - \% \\
\hline Total & $\mathbf{9 9 . 7 9 7 . 3 0 3}$ & $\mathbf{1 . 7 1 6 ( 1 1 4 . 2 7 9 )}-\mathbf{1 , 5 \%}$ \\
\hline BNT162b2 & 71.173 .035 & $2(163)-1,2 \%$ \\
\hline mRNA-1273 & 15.233 .690 & $443(31.272)-1,4 \%$ \\
\hline Ad26.COV2 & 11.544 .551 & $89(4.135)-2,2 \%$ \\
\hline ChAd0x1 & 1.846 .027 & $1.182(78.709)-1,5 \%$
\end{tabular}

Data (through September 26, 2021) summarized from: Agenzia Italiana del Farmaco. Available at: https://bi.aifa.gov.it/SASVisualAnalyticsViewer/VisualAnalyticsViewer_guest.jsp?

reportName=FVG_Intro0_report\&reportPath=/Shared+Data/BI+FARMACOVIGILANZA/Public/Report/\&appSwitcherDisabled=true (7).

Data on administered vaccine doses were retrieved from https://www.governo.it/it/cscovid19/report-vaccini/ (10)

Table 2 is available in the Supplemental Files section.

\section{Figures}

\section{Figure 1}

A patient with BRVO in the right eye with decreased visual acuity 1 week after receiving the second dose of BNT162b2. Ultrawide field color (A) image of the right eye showing retinal hemorrhage in the supero-temporal sector of the posterior pole and midperiphery. The macular OCT (B) revealed foveal edema with fluid and (C) complete resolution of the edema after theintravitreal 
injection of dexamethasone implant at 3 months. OCTA at the level of the superficial capillary plexus (D) showing non-perfusion areas in the superotemporal region and decreased vascular density; and flow damage at the level of the deep capillary plexus (E). (F) FFA showed delay in the perfusion of the supero-temporal branch vein with late leakage.

\section{Figure 2}

A patient with CRVO in the right eye with decreased visual acuity 1 week after receiving Ad26.COV2 vaccine. Ultrawide field color image $(A)$ of the right eye showing numerous flame-shaped hemorrhages in the posterior pole and in all 4 retinal quadrants and the edema of the optic disc. Early (B) and late (C) ultrawide field Fluorescein angiography images showing central retinal vein occlusion, extensive exudation and severe macular edema and optic nerve edema with hyperfluorescent disc leakage. The macular OCT (D) revealed foveal edema with intraretinal fluid and serous macular detachment. The macular OCT (E) obtained 3 months after intravitreal injection of dexamethasone showed complete resolution of the edema. (F) OCTA at the level of the superficial capillary plexus showed vascular tortuosity. G) OCTA at the level of the deep capillary plexus showed decrease in vascular perfusion.

\section{Supplementary Files}

This is a list of supplementary files associated with this preprint. Click to download.

- Table2.docx 